\title{
A Nationwide Retrospective Study of Opioid Management Patterns in 2,468 Patients with Spinal Pain in Korea
}

\author{
Sung-Soo Chung ${ }^{1}$, Chun-Kun Park ${ }^{2}$, Kyu-Jung Cho ${ }^{3}$, Kyoung Hyo Choi ${ }^{4}$, Jin-Hyok Kim ${ }^{5}$, Sung-Bum Kim ${ }^{6}$, \\ Sung-Uk Kuh ${ }^{7}$, Jae Chul Lee ${ }^{8}$, Jae Hyup Lee ${ }^{9}$, Kyu-Yeol Lee ${ }^{10}$, Sun-Ho Lee ${ }^{11}$, Seong-Hwan Moon ${ }^{12}$, \\ Si-Young Park ${ }^{13}$, Jae Hang Shim ${ }^{14}$, Byung-Chul Son ${ }^{15}$, Myung Ha Yoon ${ }^{16}$, Hye-Jeong Park ${ }^{17}$ \\ ${ }^{1}$ Department of Orthopedic Surgery, Samsung Medical Center, Sungkyunkwan University School of Medicine, Seoul, Korea \\ ${ }^{2}$ Department of Neurosurgery, Good Doctor Teun Teun Hospital, Anyang, Korea \\ ${ }^{3}$ Department of Orthopedic Surgery, Inha University Hospital, Inha University School of Medicine, Incheon, Korea \\ ${ }^{4}$ Department of Rehabilitation Medicine, Asan Medical Center, University of Ulsan College of Medicine, Seoul, Korea \\ ${ }^{5}$ Department of Orthopedic Surgery, Inje University Sanggye Paik Hospital, Inje University College of Medicine, Seoul, Korea \\ ${ }^{6}$ Department of Neurosurgery, Kyung Hee University Medical Center, Kyung Hee University School of Medicine, Seoul, Korea \\ ${ }^{7}$ Department of Neurosurgery, Gangnam Severance Hospital, Yonsei University Medical Center, Seoul, Korea \\ ${ }^{8}$ Department of Orthopedic Surgery, Soonchunhyang University Hospital, Soonchunhyang University College of Medicine, Seoul, Korea \\ ${ }^{9}$ Department of Orthopedic Surgery, SMG-SNU Boramae Medical Center, Seoul, Korea \\ ${ }^{10}$ Department of Orthopedic Surgery, Dong-A University Medical Center, Dong-A University College of Medicine, Busan, Korea \\ ${ }^{11}$ Department of Neurosurgery, Samsung Medical Center, Sungkyunkwan University School of Medicine, Seoul, Korea \\ ${ }^{12}$ Department of Orthopedic Surgery, Severance Hospital, Yonsei University Health System, Seoul, Korea \\ ${ }^{13}$ Department of Orthopedic Surgery, Korea University Anam Hospital, Korea University College of Medicine, Seoul, Korea \\ ${ }^{14}$ Department of Anesthesia and Pain Medicine, Hanyang University Guri Hospital, Hanyang University College of Medicine, Guri, Korea \\ ${ }^{15}$ Department of Neurosurgery, Seoul St. Mary's Hospital, College of Medicine, The Catholic University of Korea, Seoul, Korea \\ ${ }^{16}$ Department of Anesthesiology and Pain Medicine, Chonnam National University Hospital, \\ Chonnam National University Medical School, Gwangju, Korea \\ ${ }^{17}$ Mundipharma Korea Ltd., Seoul, Korea
}

Study Design: Retrospective patient data collection and investigator survey.

Purpose: To investigate patterns of opioid treatment for pain caused by spinal disorders in Korea.

Overview of Literature: Opioid analgesic prescription and adequacy of consumption measures in Korea have markedly increased in the past decade, suggesting changing patterns in pain management practice; however, there is lack of integrated data specific to Korean population.

Methods: Patient data were collected from medical records at 34 university hospitals in Korea. Outpatients receiving opioids for pain caused by spinal disorders were included in the study. Treatment patterns, including opioid types, doses, treatment duration, outcomes, and adverse drug reactions (ADRs), were evaluated. Investigators were interviewed on their perceptions of opioid use for spinal disorders.

Results: Among 2,468 analyzed cases, spinal stenosis (42.8\%) was the most common presentation, followed by disc herniation (24.2\%) and vertebral fracture $(17.5 \%)$. In addition, a greater proportion of patients experienced severe pain $(73.9 \%)$ rather than moderate $(19.9 \%)$ or mild $(0.7 \%)$ pain. Oxycodone $(51.9 \%)$ and fentanyl $(50.8 \%)$ were the most frequently prescribed opioids; most patients

\footnotetext{
Received Oct 27, 2016; Revised Nov 18, 2016; Accepted Nov 18, 2016

Corresponding author: Chun-Kun Park

Department of Neurosurgery, Good Doctor Teun Teun Hospital,

775 Gyeongsu-Daero Dongan-gu, Anyang 14041, Korea

Tel: +82-31-8086-8357, Fax: +82-2-534-4799, E-mail: ckpmd@catholic.ac.kr
} 
were prescribed relatively low doses. The median duration of opioid treatment was 84 days. Pain relief was superior in patients with longer treatment duration ( $\geq 2$ months) or with nociceptive pain than in those with shorter treatment duration or with neuropathic or mixed-type pain. ADRs were observed in $8.6 \%$ of cases. According to the investigators' survey, "excellent analgesic effect" was a perceived advantage of opioids, while safety concerns were a disadvantage.

Conclusions: Opioid usage patterns in patients with spinal disorders are in alignment with international guidelines for spinal pain management. Future prospective studies may address the suitability of opioids for spinal pain treatment by using appropriate objective measurement tools.

Keywords: Spine; Spinal diseases; Chronic pain; Opioid

\section{Introduction}

Spine and back pain are among the most common sources of chronic noncancer pain among adults. Low back pain is a globally prevalent concern, with an estimated lifetime prevalence of approximately 40\% [1]. The UK National Institute for Health and Care Excellence and the American College of Physicians/American Pain Society (ACP/ APS) guidelines provide recommendations for managing chronic low back pain through various pharmacological and nonpharmacological treatment modalities $[2,3]$. Despite the variety of treatment modalities in use, a considerable amount of needs in chronic pain management remain unmet. For example, pain-relieving medications were estimated to be the most commonly prescribed pharmacotherapy in ambulatory care in the United States (US) [4].

Pharmacotherapies recommended by the ACP/APS guidelines for subacute and chronic back pain include acetaminophen, benzodiazepine, tramadol, and opioids [2]. Opioids are considered effective for reducing nociceptive pain and are moderately effective for neuropathic pain; however, tolerability issues such as nausea and vomiting tend to limit their application in clinical practice. Despite these limitations, opioids can be both effective and safe, if appropriately used $[3,5]$.

Long-term use of opioids for chronic noncancer pain remains controversial owing to concerns regarding analgesic tolerance and drug dependence [6-10]. The ratio of opioid to total analgesic use in Korea is still relatively low compared with Western countries. In a study evaluating the adequacy of opioid use at the national level with the Adequacy of Consumption Measure (ACM), Korea was listed with a $47.0 \%$ adequacy score for opioid con- sumption in 2010 [11]. This implies that less than half of the patients in Korea who required opioid treatment actually received it. In 2010, the adequacy scores of other countries were as follows: USA, 229.7\%; Canada, 312.6\%; France, 73.5\%; UK, 66.4\%; Japan, 15.5\%; and China, 1.2\% [11]. This indirectly indicates limited usage of opioids for management of spinal pain in Korea.

Nevertheless, opioid usage in Korea is growing rapidly. The adequacy level of opioid consumption, as indicated by the ACM, has increased by over six-fold during the 20062010 period $[11,12]$. Such a remarkable improvement suggests changing attitudes toward pain management [9]. Considering the lack of integrated data specific to the Korean population, we sought to better understand the effectiveness and safety of opioid use in the management of different types of spinal pain arising from multiple causes $[5,13]$.

This retrospective study was designed to investigate the following: (1) patterns of opioid usage in patients with pain caused by spinal disorders; (2) physicians' perceptions on opioid usage; and (3) opioid analgesic usage for spinal disorders.

\section{Materials and Methods}

\section{Patients}

Since this study was a retrospective study, the Institutional Review Boards of the 34 participating hospitals approved the study and granted waiver for collecting informed consent from patients. All information was anonymized in order to maintain patient confidentiality. Data were collected from medical records of 34 university hospitals in Korea. The list of patients was acquired from August 2011 
database, which included patients who visited hospitals due to spinal disorders in November 2010 or earlier, with an aim to collect data of more than 50 patients per investigator. In outpatient clinics with $<50$ patients in November 2010, patients from the preceding month were included until the number reached 50. The investigators reviewed patient medical records and extracted information including demographics, diagnoses, type and dose of the prescribed opioids, and comorbidities. Korean outpatients of both sexes, aged $\geq 20$ years, who received opioids at least once to treat pain arising from spinal diseases in November 2010 or earlier were eligible for inclusion.

Exclusion criteria were used to eliminate cases wherein external factors may have influenced clinical decisions regarding opioid usage. Any patient participating in an interventional study during the study period was excluded. In addition, patients with cancer-related pain caused by primary or metastatic spinal cancer were excluded. In order to minimize selection bias, 50 consecutive cases were enrolled per investigator from the patients who met the selection criteria.

\section{Definitions}

Spinal disorders assessed in this study included, but were not limited to, spinal stenosis, disc herniation, vertebral fracture, persistent postoperative back pain, unspecified back pain, intervertebral disc disorders, spondylolisthesis, spinal cord injury, and spinal infection. Diagnosis was considered as a multiple-option item in the case report form because the medical records often described more than one spinal condition per patient.

Opioid analgesics included, but were not limited to, oxycodone (immediate release [IR], prolonged release $[\mathrm{PR}]$, and other composite formulations), fentanyl, hydromorphone, and codeine in combination with acetaminophen and ibuprofen. These commercially available medications are classified as "narcotics" by the Act on the Control of Narcotics, the Enforcement of Decree of the Act on the Control of Narcotics, and other relevant laws in Korea. A codeine combination is classified as a narcotic in Korea, but codeine is otherwise classified as a weak opioid according to the analgesic ladder published by the World Health Organization [14].

Pain intensity was recorded using a numeric rating scale (NRS $[15,16])$ where available. NRS scores were converted into following categories: "severe pain" for scores 7-10, "moderate pain" for scores 4-6, and "mild pain" for scores $1-3$. In cases where no NRS values were recorded, pain intensity was assessed as severe, moderate, or mild according to the descriptions in the medical records. Pain intensity was examined at the initiation of opioid treatment and 1 and 2 months after treatment initiation. Moreover, adverse drug reactions (ADRs) after opioid administration were recorded.

\section{Investigators' questionnaire}

Participating investigators and subinvestigators were interviewed to assess their perceptions regarding the usage of opioid analgesics. Investigators were also asked about their concerns regarding opioid dependency and their experiences with dependent patients. In addition, they were asked to suggest actions or measures that may improve opioid usage in Korea.

\section{Statistical analyses}

Descriptive statistics were used to summarize patient characteristics and opioid treatment patterns. Means and standard deviations or medians and ranges were used for continuous data. Categorical data were presented as numbers and percentages. The number of cases and the percentages of ADRs were presented as well. Results of the investigators' questionnaire were descriptively summarized.

Pearson's chi-square test was used to identify statistically significant relationships (1) between type of pain experienced and presence/absence of pain relief with opioid treatment and (2) between duration of opioid treatment and presence/absence of pain relief. All reported $p$-values were two sided; $p$-values $<0.05$ were considered significant. Statistical analyses were performed using SAS ver. 9.1 (SAS Institute, Cary, NC, USA).

\section{Results}

\section{Baseline characteristics}

In total, data regarding 2,483 cases were collected from 34 hospitals. Of these, 2,468 cases were analyzed after excluding 15 cases with protocol violations. Table 1 summarizes patient characteristics. The study population contained more female $(60.0 \%)$ than male $(40.0 \%)$ patients, and 
Table 1. Patient characteristics $(n=2,468)$

\begin{tabular}{|c|c|}
\hline Characteristic & No. $(\%)$ \\
\hline \multicolumn{2}{|l|}{ Demographic information } \\
\hline Age (mean $\pm S D)$, yr & $64.2 \pm 14.1$ \\
\hline \multicolumn{2}{|l|}{ Sex } \\
\hline Male & $986(40.0)$ \\
\hline Female & $1,482(60.0)$ \\
\hline \multicolumn{2}{|l|}{ Diagnosis (multiple diagnoses possible) } \\
\hline Spinal stenosis & $1,056(42.8)$ \\
\hline Disc herniation & $597(24.2)$ \\
\hline Vertebral fracture & $431(17.5)$ \\
\hline Persistent postoperative back pain & $320(13.0)$ \\
\hline Unspecified back pain & $264(10.7)$ \\
\hline Intervertebral disc disorders & $211(8.6)$ \\
\hline Spondylolisthesis & $204(8.3)$ \\
\hline Spinal cord injury & $100(4.1)$ \\
\hline Spinal infection & $46(1.9)$ \\
\hline Others & $457(18.5)$ \\
\hline \multicolumn{2}{|l|}{ Type of pain } \\
\hline Neuropathic pain only & $694(28.1)$ \\
\hline Nociceptive pain only & $635(25.7)$ \\
\hline Both neuropathic and nociceptive pain & $1,027(41.6)$ \\
\hline No information & $112(4.5)$ \\
\hline \multicolumn{2}{|l|}{$\begin{array}{l}\text { Pain intensity at the initiation of } \\
\text { opioid treatment }\end{array}$} \\
\hline Severe & $1,823(73.9)$ \\
\hline Moderate & 490 (19.9) \\
\hline Mild & $18(0.7)$ \\
\hline No information & $137(5.6)$ \\
\hline \multicolumn{2}{|l|}{$\begin{array}{l}\text { Duration of pain prior to the initiation } \\
\text { of opioid treatment }\end{array}$} \\
\hline$<3 \mathrm{mo}$ & $865(35.0)$ \\
\hline 3 to 12 mo & $473(19.2)$ \\
\hline$\geq 12 \mathrm{mo}$ & $962(39.0)$ \\
\hline No information & $168(6.8)$ \\
\hline
\end{tabular}

SD, standard deviation.

the mean age was 64.2 years. Spinal stenosis $(42.8 \%)$ was the most common diagnosis, followed by disc herniation (24.2\%), vertebral fracture (17.5\%), persistent postoperative back pain (13.0\%), and unspecified back pain (10.7\%).

Of the 2,468 patients analyzed, 694 (28.1\%), 635 (25.7 $\%)$, and 1,027 (41.6\%) patients reported neuropathic, nociceptive, and mixed (both neuropathic and nociceptive) pain, respectively. Before initiating opioid therapy, 1,823 (73.9\%) patients experienced severe pain, 490 (19.9\%) experienced moderate pain, whereas $18(0.7 \%)$ experienced mild pain. Only 593 (24.0\%) patients underwent an NRS assessment of pain intensity; the mean pain score was 7.5. The results majorly indicated severe pain levels in the study population.

The duration of pain before initiating opioid treatment was $<3$ months in 865 (35.0\%) patients, 3-12 months in 473 (19.2\%) patients, and $\geq 12$ months in 962 (39.0\%) patients (Table 1).

\section{Nonopioid analgesia before initiation of opioid treatment}

Over half of the patients $(1,389 / 2,468 ; 56.3 \%)$ were treated with nonopioid analgesics before receiving opioids for spinal pain. Of these, 847 (34.3\%) patients had received weak opioids (including tramadol), 639 (25.9\%) patients had received nonsteroidal anti-inflammatory drugs (NSAIDs), and 264 (10.7\%) patients had received anticonvulsants.

\section{Opioid treatment patterns}

Prescriptions were classified as regular or pro re nata (PRN). In 2,060 (83.5\%) patients, opioid therapy was administered regularly, whereas in $408(16.5 \%)$ patients, it was prescribed only when required. The opioid types prescribed, daily doses, and treatment duration for each opioid analgesic are summarized in Table 2. Oxycodone (PR, IR, and other composite drugs combined) was the most commonly prescribed drug (in 51.9\% patients), followed by fentanyl (50.8\%) and codeine combinations (20.0\%). The median daily dose of fentanyl was $12.0 \mu \mathrm{g} /$ $\mathrm{hr}$ (range, 10.0-75.0 $\mu \mathrm{g} / \mathrm{hr}$ ), while the median daily doses of oxycodone PR and IR were $20.0 \mathrm{mg}$ (range, 5.0-200.0 $\mathrm{mg}$ ) and $10.0 \mathrm{mg}$ (range, $5.0-60.0 \mathrm{mg}$ ), respectively. The median daily dose of codeine combinations was $30.0 \mathrm{mg}$ (range, 10.0-80.0 mg). The patients in this study had received relatively low-dose opioid prescriptions according to the recommendations of the Korean Ministry of Health and Welfare's 2012 Cancer Pain Treatment Guideline [17]. The median treatment duration of fentanyl, oxycodone PR, and codeine combinations was 67, 120, and 46 days, respectively. 
Table 2. Opioid types, dosages and treatment durations $\left(n=2,395^{a}\right)$

\begin{tabular}{|c|c|c|c|c|c|}
\hline \multirow{2}{*}{ Opioid type } & \multirow{2}{*}{ No. $(\%)$} & \multicolumn{2}{|c|}{ Daily dose } & \multicolumn{2}{|c|}{ Duration of treatment (day) } \\
\hline & & Mean \pm SD & Median (range) & Mean \pm SD & Median (range) \\
\hline Overall & $2,395(100.0)$ & - & - & $272.6 \pm 401.9$ & $84.0(1.0,6,148.0)$ \\
\hline Oxycodone & $1,242(51.9)$ & - & - & - & - \\
\hline PR & $779(32.5)$ & $26.2 \pm 15.9 \mathrm{mg}$ & $20.0(5.0,200.0)$ & $282.6 \pm 398.1$ & $120.0(1.0,2966.0)$ \\
\hline $\mathbb{R}$ & $449(18.7)$ & $12.8 \pm 8.0 \mathrm{mg}$ & $10.0(5.0,60.0)$ & $147.7 \pm 273.8$ & $36.0(1.0,2377.0)$ \\
\hline Oxycodone (others) ${ }^{b)}$ & $14(0.6)$ & $13.9 \pm 2.1 \mathrm{mg}$ & $15.0(10.0,15.0)$ & $542.9 \pm 571.7$ & $215.0(12.0,1447.0)$ \\
\hline Fentanylc) $^{c}$ & $1,217(50.8)$ & $17.6 \pm 8.9 \mu \mathrm{g} / \mathrm{hr}$ & $12.0(10.0,75.0)$ & $252.1 \pm 348.9$ & $67.0(1.0,2,161.0)$ \\
\hline Codeine combination $^{\mathrm{d} l}$ & $479(20.0)$ & $32.6 \pm 14.0 \mathrm{mg}$ & $30.0(10.0,80.0)$ & $186.3 \pm 376.4$ & $46.0(1.0,5551.0)$ \\
\hline Hydromorphone & $115(4.8)$ & $8.9 \pm 4.3 \mathrm{mg}$ & $8.0(2.0,30.0)$ & $94.2 \pm 104.8$ & $57.0(1.0,709.0)$ \\
\hline Morphine & $64(2.7)$ & $34.7 \pm 26.1 \mathrm{mg}$ & $20.0(10.0,120.0)$ & $209.0 \pm 443.1$ & $31.0(2.0,2556.0)$ \\
\hline Hydrocodone & $14(0.6)$ & $21.5 \pm 2.6 \mathrm{mg}$ & $22.5(15.0,22.5)$ & $103.1 \pm 142.4$ & $29.0(8.0,464.0)$ \\
\hline
\end{tabular}

SD, standard deviation; PR, prolonged release; IR, immediate release.

a) Data on the type of opioids, dosages and treatment durations were not obtainable from 73 patients; ${ }^{\text {b) } O x y c o d o n e ~(o t h e r s) ~ i n d i c a t e s ~ c o m p o s i t e ~ d r u g s ; ~}$

${ }^{c}$ Units for fentanyl are expressed as $\mu \mathrm{g} / \mathrm{hr} ;{ }^{d)}$ The dose refers to the quantity of codeine in the codeine combination.

Table 3. Proportion of patients experiencing pain relief with opioid analgesic treatment by type of pain $(n=1,945)$

\begin{tabular}{|c|c|c|c|}
\hline \multirow{2}{*}{ Type of pain } & \multirow{2}{*}{ No. } & \multicolumn{2}{|c|}{ Experienced pain relief with opioid analgesic treatment ${ }^{a)}$} \\
\hline & & Yes & No \\
\hline Overall & $1,945^{b)}$ & $1,055(54.2)$ & $890(45.8)$ \\
\hline Nociceptive pain only & 504 & $319(63.3)^{c}$ & $185(36.7)^{c)}$ \\
\hline Neuropathic pain only & 620 & $350(56.5)^{c /}$ & $270(43.5)^{c)}$ \\
\hline Both neuropathic and nociceptive pain & 821 & $386(47.0)^{c)}$ & $435(53.0)^{c)}$ \\
\hline
\end{tabular}

Values presented in parentheses are \%.

a)Pearson's chi-square test was used to test for statistically significant association between type of pain and experience of pain relief with treatment (Yes/No) $(p=0.001)$; ${ }^{\text {b) }}$ Data on the type of pain and experience of pain relief (Yes/No) were not obtainable for 523 patients; ${ }^{\text {c) }}$ Percentages given in brackets indicate the proportion that experienced pain relief (Yes/No) for each type of pain.

\section{Opioid treatment outcomes and relationships with pain type and treatment duration}

Achievement of pain relief with opioid treatment was significantly related to the type of pain. The proportion of patients experiencing pain relief differed across the three types of pain, and the difference was significant $(p<0.0001)$ (Table 3). The proportion of patients who experienced pain relief was highest in the group with nociceptive pain alone $(63.3 \%)$, followed by that with neuropathic pain alone $(56.5 \%)$ and that with mixed-type pain $(47.0 \%)$ (Table 3).

Moreover, achievement of pain relief was significantly related to opioid treatment duration $(<1$ month, 1-2 months, and $\geq 2$ months); statistically significant differ- ences were noted across all three treatment duration categories $(p=0.0013)$ (Table 4$)$. In comparison with shorter treatment durations, treatment duration of $\geq 2$ months resulted in a greater proportion of patients experiencing pain relief (Table 4).

\section{Concomitant treatment for spinal pain management}

Of the 2,468 patients, 1,467 (59.4\%) patients received concomitant treatment with surgery, physical therapy, or nonsurgical interventions for pain control in addition to opioid therapy. Concomitant analgesics were prescribed in $1,513(61.3 \%)$ patients. The most frequently prescribed concomitant drug was tramadol in 779 (31.6\%) patients, followed by NSAIDs in 685 (27.8\%) patients and anticon- 
Table 4. Proportion of patients experiencing pain relief with opioid analgesic treatment by duration of treatment $(n=2,037)$

\begin{tabular}{|c|c|c|c|}
\hline \multirow{2}{*}{ Duration of treatment } & \multirow{2}{*}{ No. } & \multicolumn{2}{|c|}{ Experienced pain relief following opioid analgesics administration ${ }^{\text {a) }}$} \\
\hline & & Yes & No \\
\hline Overall & $2,037^{b /}$ & $1,087(53.4)$ & $950(46.6)$ \\
\hline$<1 \mathrm{mo}$ & 611 & $296(48.5)^{c)}$ & $315(51.6)^{c)}$ \\
\hline $1-2 \mathrm{mo}$ & 254 & $125(49.2)^{c}$ & $129(50.8)^{c l}$ \\
\hline$\geq 2 \mathrm{mo}$ & 1,172 & $666(56.8)^{c)}$ & $506(43.2)^{c)}$ \\
\hline
\end{tabular}

Values presented in parentheses are \%.

alPearson's chi-square test was used to test for statistically significant association between opioid therapy duration and experience of relief (Yes/ No) $(p=0.013)$; ${ }^{b}$ Data on the duration of opioid therapy and experience of pain relief (Yes/No) were not obtainable for 431 patients; ${ }^{\text {cl }}$ Percentages given in brackets indicate the proportion experiencing pain relief (Yes/No) for each treatment duration category.

Table 5. Concomitant treatment for management of spinal pain in addition to opioid treatment $(n=2,468)$

\begin{tabular}{lr} 
Category (details) & No. (\%) \\
\hline Concomitant non-pharmacological therapy for management of spinal pain other than opioid treatment & $1,467(59.4)$ \\
\hline Yes & 927 (37.6) \\
\hline Non-surgical intervention & 521 (21.1) \\
\hline Surgical treatment & 439 (17.8) \\
\hline Physical therapy & 53 (2.1) \\
\hline Chinese medicine (acupuncture, moxibustion, & 100 (4.1) \\
\hline heat/ice pack, herbal medicine) & 897 (36.4) \\
\hline Others & 104 (4.2) \\
\hline No & 1,513 (61.3) \\
\hline No information & $779(31.6)$ \\
\hline Concomitant pharmacotherapy for management of spinal pain besides opioid treatment & 685 (27.8) \\
\hline Yes & $389(15.8)$ \\
\hline Tramadol and tramadol Combinations & 404 (16.4) \\
\hline Non-steroidal anti-inflammatory drugs & 955 (38.7) \\
\hline Anticonvulsants & 0 (0.0) \\
\hline Others & \\
\hline No information & \\
\hline
\end{tabular}

NSAIDs, non-steroidal anti-inflammatory drugs.

vulsants in 389 (15.8\%) patients (Table 5).

\section{Investigator survey results}

Investigators' perceptions regarding opioid treatment for spinal disorders are presented in Table 6. In total, 75 investigators and subinvestigators responded to the survey. Of these respondents, 30 (40.0\%) had expertise in orthopedics, $25(33.3 \%)$ in neurosurgery, $18(24.0 \%)$ in anesthesiology and pain medicine, and $2(2.7 \%)$ in rehabilitation medicine.

Among these, 72 (96.0\%) respondents perceived "excellent analgesic effect" as the primary advantage of opioid analgesics in outpatient clinics; 50 (66.7\%) investigators responded that "concern for early side effects" was a disadvantage of opioid analgesics. "Long-term safety concerns including drug dependence" were chosen by 34 (45.3\%) respondents, and 60 (80.0\%) investigators had experienced patients with opioid dependence; however, most $(81.7 \%)$ investigators answered that true cases of 
Table 6. Investigators' perceptions and suggestions regarding opioid analgesic use in Korea $(\mathrm{n}=75)$

\begin{tabular}{|c|c|}
\hline Category (details) & No. $(\%)^{a)}$ \\
\hline \multicolumn{2}{|l|}{ Perceived advantages of opioid analgesic prescription in outpatient clinics } \\
\hline Excellent analgesic effect & $72(96.0)$ \\
\hline Useful for controlling persistent pain following surgery & $42(56.0)$ \\
\hline Useful for patients in whom surgery is prohibitively difficult & $36(48.0)$ \\
\hline Useful as a conservative preoperative treatment & $20(26.7)$ \\
\hline Others & $1(1.3)$ \\
\hline \multicolumn{2}{|l|}{ Perceived drawbacks of opioid analgesic prescription in outpatient clinics } \\
\hline Concerns for early side effects & $50(66.7)$ \\
\hline Long-term safety concerns, including drug dependence & $34(45.3)$ \\
\hline Ongoing side effect concerns & $33(44.0)$ \\
\hline Short reimbursement period & $21(28.0)$ \\
\hline Complex administrative processes including issuance of opioid prescription & $19(25.3)$ \\
\hline Burdensome procedure required for explanation to patients & $18(24.0)$ \\
\hline Others & $2(2.7)$ \\
\hline \multicolumn{2}{|l|}{ Suggestions for better opioid analgesic use } \\
\hline Development of drugs with fewer side effects and easier titration & $57(76.0)$ \\
\hline Development of guidelines and better clinical data applicable to Koreans & $53(70.7)$ \\
\hline Better administrative conditions including reimbursement and regulations & $38(50.7)$ \\
\hline Meetings for idea exchange and education regarding analgesic use & $28(37.3)$ \\
\hline Others & $1(1.3)$ \\
\hline
\end{tabular}

${ }^{\text {al }}$ Respondents comprised 75 principal investigators and sub-investigators participating in this study. Areas of expertise include Orthopaedics, Neurosurgery, Anaesthesiology and Pain Medicine, and Rehabilitation Medicine.

Table 7. Incidence of adverse drug reactions $(n=2,468)$

\begin{tabular}{|cc|}
\hline Adverse drug reaction (ADR) ${ }^{a)}$ & No. (\%) \\
\hline Total number of patients with ADRs & $211(8.6)$ \\
\hline Vomiting & $85(3.4)$ \\
\hline Constipation & $64(2.6)$ \\
\hline Dizziness & $30(1.2)$ \\
\hline Somnolence & $15(0.6)$ \\
\hline Pruritus & $12(0.5)$ \\
\hline Gastrointestinal disorder & $7(0.3)$ \\
\hline Dyspepsia & $5(0.2)$ \\
\hline Abdominal discomfort & $3(0.1)$ \\
\hline Headache & $3(0.1)$ \\
\hline Decreased appetite & $3(0.1)$ \\
\hline
\end{tabular}

${ }^{a)} A D R s$ considered as possibly related to the administration of opioid analgesics are shown in the table above. Multiple ADRs could be recorded for individual patients. Only the 10 most frequently reported ADRs are listed. drug dependence were rarely observed.

For improved use of opioid analgesics, a majority of investigators suggested solutions such as "development of drugs with fewer side effects and easier titration" (76.0\%) and "guidelines and clinical data applicable to the Korean population" (70.7\%).

\section{Adverse drug reactions}

Of the 2,468 patients eligible for analysis, 211 patients (of 2,468 [8.6\%] patients, 259 cases) experienced ADRs wherein opioid analgesic-related causality could not be ruled out. ADR severity was mild in $49.7 \%$ (157/259) cases, moderate in $21.2 \%$ (67/259) cases, and severe in $11.1 \%$ (35/259) cases. The three most frequently reported ADRs were vomiting, constipation, and dizziness (Table 7).

\section{Discussion}

This study aimed to provide an original integrated set of 
data on the issue of opioid treatment practice for spinal pain in Korea. Through a nationwide investigation to observe treatment patterns across the country, this study achieved its primary goals with a large cohort of 2468 patients.

The management of spinal pain requires active treatment plans and implementation. Recommended treatment modalities for spinal pain management can be classified into four categories: interventional pain therapies, complex medication (including opioids and neuropathic pain medications), high-intensity cognitive behavioral therapy-based programs, and spinal surgery [18]. Despite various treatment modalities, there has been very limited systematic research to guide and improve the use of opioid analgesics for managing spinal disorder-associated pain. This may be a reason for conservative opioid prescription practices. For example, an observational study that examined opioid use for spinal and radicular pain in 25,479 patients revealed that only $3.4 \%$ (867) patients were treated with opioids [6].

In a manual guiding the use of opioids for persistent pain, the British Pain Society (BPS) recommends strict restrictions on the use of strong opioids [19]. The guidelines state that (1) opioids should not be used as a firstline treatment, (2) patients should be adequately informed about the long-term effects of opioids, including the possibility of adverse events, (3) mental health history should be recorded before treatment to aid exclusion of patients with depression or substance misuse disorders, and (4) whenever possible, modified-release opioid formulations at regular intervals should be used. In addition, the BPS guidelines for low back pain recommend that the lowest dose possible should be used for the shortest time possible $[18,19]$. Other recent guidelines and physician resources for opioid prescription, such as guidelines from the Centers for Disease Control and Prevention and the UK Royal College of Anaesthetists, state that opioids are not effective for long-term use $[20,21]$.

Our results indicate that the current prescribing practice in Korea is generally in alignment with the BPS guidelines in terms of opioid dosage. Most cases in our study were prescribed daily doses of opioids that were in the lowest range recommended in the Korean Ministry of Health and Welfare's Cancer Pain Treatment Guidelines [17]; however, our data also revealed that $50 \%$ of patients had received opioid analgesics for $>3$ months, which may be attributed to the lack of adequate treatment options for chronic pain.

This study indicates that opioid analgesics are a more appropriate treatment option for nociceptive pain than for neuropathic or mixed-type pain. In general, opioids are known to be excellent pain relievers, particularly for nociceptive pain. In addition, higher opioid doses are needed more often for treatment of neuropathic pain than for nociceptive pain [22]. Therefore, the results from this study are in agreement with the existing knowledge; however, we note that our observations regarding the type of pain could be alternatively interpreted, that is, patients with neuropathic or mixed-type pain may generally not have had the opportunity to receive more optimal treatment with opioids or concomitant adjuvants to reach adequate levels of pain control.

To the best of our knowledge, there are no local reports dedicated to physician surveys investigating opioid usage for managing spinal pain. The results of our investigators' survey suggest that Korean physicians adequately understand the efficacy parameters of opioid usage; however, they are concerned regarding the safety issues such as addiction although the ADRs from opioid usage in this study were limited to $8.6 \%$, and there were no reports of actual addiction. These concerns may contribute to the modest opioid usage index in Korea, which is still below $50 \%$ [22]. Although the guidelines for use of opioids in cancer pain management are relatively well established [17] and adopted in clinical practice in Korea, use of opioids for noncancer pain treatment (including spinal disorders) is estimated to be much lower. As indicated by the investigators' survey responses, there is need for more local clinical data and development of improved guidelines for use of opioids to treat spinal pain in Korea.

The NRS is a well-validated and preferred unidimensional self-report tool for pain assessment [23,24]; thus, it should be used more widely in clinical settings. Nevertheless, in this study, NRS pain assessments were only recorded for $24.0 \%$ of the 2,468 patients. Because the suitability of pain alleviation by opioid therapy cannot be fully supported or rejected unless appropriate, standardized methods for pain measurement are applied [5,13,15,16,25-27]. Our data imply that additional education is needed on regular assessment and documentation of pain in clinical practice.

We note several limitations inherent to the nature of the study. First, we only assessed patients who received opioid treatment for spinal disorders and did not examine the 
entire spinal disorder population. Thus, it is difficult to analyze global factors that affect clinical decisions related to spinal pain management, for example, the data collected do not permit us to explain how clinical decisions were made on whether to prescribe opioid analgesics or to choose other treatment modalities. Second, certain patients were receiving ongoing opioid treatment at the time of data collection and had not yet completed the full treatment course. For such patients, data for the entire treatment duration and ultimate outcomes of opioid treatment were not available at the point of data collection. Third, reporting of adverse events or ADRs may have been incomplete owing to the retrospective nature of the study.

\section{Conclusions}

To the best of our knowledge, this is the first large-scale report on patterns of opioid treatment in 2468 patients with spinal disorders in university hospitals across Korea. The findings demonstrated an overall alignment of current practices in Korea with international guidelines for opioid-based management of spinal pain. In particular, the results may be a valuable local source of preliminary data for treatment guidelines on spinal disorders in Korea. The findings underscore the importance of objective measurements and appropriate documentation of pain to guide opioid usage for management of spinal pain in Korea.

\section{Conflict of Interest}

Hye-Jeong Park is an employee of Mundipharma Korea Ltd. All other authors have no relevant conflicts of interest to declare.

\section{Acknowledgments}

This study was sponsored by Mundipharma Korea Ltd.

We would like to thank the following investigators for their contributions to the study: Jae-Sung Ahn, KiChan An, Hyun-Chul Choi, Sang-Sik Choi, Seung-Won Choi, Tai-Hyong Cho, Chun-Kee Chung, Ho-Gyun Ha, Changju Hwang, Soo-Bin Im, Sang-Ryong Jeon, Ju-Ho Jeong, Chang-Nam Kang, Dae-Hyun Kim, Dong-Hee Kim, Dong-Soo Kim, Eung-Ha Kim, Keung-Nyun Kim, Sang-Hyun Kim, Se-Hoon Kim, Seok-Woo Kim, SangBong Ko, Ki-Hyung Koo, Kwang-Bok Lee, Jung-Hee Lee,
Sang-Eun Lee, Woo-Yong Lee, Francis Sahngun Nahm, Kun-Woo Park, Jin-Woo Shin, Sung-Eun Sim, Geun-Sung Song, Sang Hoon Yoon and Seung Hwan Yoon.

\section{References}

1. Hoy D, Bain C, Williams G, et al. A systematic review of the global prevalence of low back pain. Arthritis Rheum 2012;64:2028-37.

2. Chou R, Qaseem A, Snow V, et al. Diagnosis and treatment of low back pain: a joint clinical practice guideline from the American College of Physicians and the American Pain Society. Ann Intern Med 2007; 147:478-91.

3. National Institute for Health and Clinical Excellence. Low back pain in adults: early management. Clinical guideline CG88 2009 [Internet]. London: National Institute for Health and Care Excellence: c2016 [cited 2016 Oct 10]. Available from: https://www.nice.org. uk/guidance/cg88/resources/low-back-pain-inadults-early-management-975695607493.

4. Raofi S, Schappert SM. Medication therapy in ambulatory medical care: United States, 2003-04. Vital Health Stat 13 2006;(163):1-40.

5. Morlion B. Pharmacotherapy of low back pain: targeting nociceptive and neuropathic pain components. Curr Med Res Opin 2010;27:11-33.

6. Fanciullo GJ, Ball PA, Girault G, Rose RJ, Hanscom B, Weinstein JN. An observational study on the prevalence and pattern of opioid use in 25,479 patients with spine and radicular pain. Spine 2002;27:201-5.

7. Gadzhanova S, Bell JS, Roughead EE. What analgesics do older people use prior to initiating oxycodone for non-cancer pain? A retrospective database study. Drugs Aging 2013;30:921-6.

8. Pergolizzi J, Boger RH, Budd K, et al. Opioids and the management of chronic severe pain in the elderly: consensus statement of an International Expert Panel with focus on the six clinically most often used World Health Organization Step III opioids (buprenorphine, fentanyl, hydromorphone, methadone, morphine, oxycodone). Pain Pract 2008;8:287-313.

9. Vallerand A, Nowak L. Chronic opioid therapy for nonmalignant pain: the patient's perspective. Part I-life before and after opioid therapy. Pain Manag Nurs 2009;10:165-72.

10. van Ojik AL, Jansen PA, Brouwers JR, van Roon EN. 
Treatment of chronic pain in older people: evidencebased choice of strong-acting opioids. Drugs Aging 2012;29:615-25.

11. Duthey B, Scholten W. Adequacy of opioid analgesic consumption at country, global, and regional levels in 2010, its relationship with development level, and changes compared with 2006. J Pain Symptom Manage 2014;47:283-97.

12. Seya MJ, Gelders SF, Achara OU, Milani B, Scholten WK. A first comparison between the consumption of and the need for opioid analgesics at country, regional, and global levels. J Pain Palliat Care Pharmacother 2011;25:6-18.

13. Fine PG, Mahajan G, McPherson ML. Long-acting opioids and short-acting opioids: appropriate use in chronic pain management. Pain Med 2009;10 Suppl 2:S79-88.

14. Best Practice Advocacy Centre New Zealand. WHO Analgesic Ladder: which opioid to use at step two? Best Pract J 2008;(18):20-3.

15. Breivik H, Cherny N, Collett B, et al. Cancer-related pain: a pan-European survey of prevalence, treatment, and patient attitudes. Ann Oncol 2009;20:142033.

16. Breivik H, Collett B, Ventafridda V, Cohen R, Gallacher D. Survey of chronic pain in Europe: prevalence, impact on daily life, and treatment. Eur J Pain 2006;10:287-333.

17. Korean Ministry of Health and Welfare. Cancer pain management guideline. 5th ed. Seoul: Korean Ministry of Health and Welfare; 2012.

18. Lee J, Gupta S, Price C, Baranowski AP; British Pain Society. Low back and radicular pain: a pathway for care developed by the British Pain Society. Br J Anaesth 2013;111:112-20.
19. British Pain Society. Opioids for persistent pain: summary of guidance on good practice from the British Pain Society. Br J Pain 2012;6:9-10.

20. Dowell D, Haegerich TM, Chou R. CDC Guideline for Prescribing Opioids for Chronic Pain: United States, 2016. JAMA 2016;315:1624-45.

21. Royal College of Anaesthetists Faculty of Pain Medicine. Opioids Aware: A resource for patients and healthcare professionals to support prescribing of opioid medicines for pain [Internet]. London: The Royal College of Anaesthetists; [cited 2016 Oct 10]. Available from: https://www.fpm.ac.uk/faculty-ofpain-medicine/opioids-aware.

22. Ballantyne JC. Opioid analgesia: perspectives on right use and utility. Pain Physician 2007;10:479-91.

23. Briggs M, Closs JS. A descriptive study of the use of visual analogue scales and verbal rating scales for the assessment of postoperative pain in orthopedic patients. J Pain Symptom Manage 1999;18:438-46.

24. Fosnocht DE, Chapman CR, Swanson ER, Donaldson GW. Correlation of change in visual analog scale with pain relief in the ED. Am J Emerg Med 2005;23: 55-9.

25. Breuer B, Fleishman SB, Cruciani RA, Portenoy RK. Medical oncologists' attitudes and practice in cancer pain management: a national survey. J Clin Oncol 2011;29:4769-75.

26. Gourlay DL, Heit HA, Almahrezi A. Universal precautions in pain medicine: a rational approach to the treatment of chronic pain. Pain Med 2005;6:107-12.

27. Silvoniemi M, Vasankari T, Vahlberg T, Vuorinen E, Clemens KE, Salminen E. Physicians' self-assessment of cancer pain treatment skills: more training required. Support Care Cancer 2012;20:2747-53. 